\title{
Detectability of motion as a factor in depth perception by monocular movement parallax
}

\author{
WOLFGANG HELL and ROBERT B. FREEMAN, JR. \\ Universität Konstanz, 7750 Konstanz, West Germany
}

\begin{abstract}
A display of two objects at different distances was presented to 10 observers, who were requested in two experiments to match the width of the more distant (comparison) object to the width of the nearer (standard) one under conditions permitting monocular observation and lateral head motion. The matched width of the comparison object was considered a measure of the effectiveness of movement parallax. The effectiveness of movement parallax decreases with increasing angular separation of the objects and with increasing background distance. A background without visible texture leads to a better perception of depth between two objects than a textured background: The results can be explained by postulating that, whenever the detectability of motion is enhanced, i.e., the threshold for the detection of motion is lowered, the effectiveness of movement parallax as a cue to depth is increased.
\end{abstract}

While passive monocular movement parallax was investigated in early studies at the threshold level (Graham, Baker, Hecht, \& Lloyd, 1948; Zegers, $1948)$ as well as under suprathreshold conditions (Gibson, Gibson, Smith, \& Flock, 1959; Smith \& Smith, 1963), monocular movement parallax has been studied more recently under conditions permitting active head motion. Ferris (1972) and Eriksson (Note 1), using a distance estimation method, concluded that monocular movement parallax was a cue to absolute depth, and Johansson (1973), using a distance matching method, regarded monocular movement parallax as a main cue to veridical monocular near-space $(<2 \mathrm{~m})$ perception. On the other hand, Gogel and Tietz $(1973,1974)$, whose subjects indicated the direction and magnitude of the apparent motion of stationary objects with head motion, reported "a failure of the cue of motion parallax to determine perceived distance" (Gogel \& Tietz, 1973, p. 291) under their experimental conditions.

The present study investigates the influence on the effectiveness of movement parallax (1) of lateral separation and (2) of the motion contrast between the objects and the background. Size matching is used to determine the effectiveness of movement parallax as a cue to depth, a method new to movement parallax research.

The influence of the angular separation upon the effectiveness of movement parallax was investigated in the first experiment. Zegers (1948) had found that the threshold angular velocity between two moving

The research reported in this article was supported by Graduate Fellowship No. 15/00183 from the Federal Republic of Germany to the first author and by Research Grant No. 33/73 from the University of Konstanz to the second author. needles was dependent of the angular "offset." Freeman (1970), in a general study, suggested that the cue effectiveness (E) depended not only on the geometrical properties of cues but also on their absolute threshold $\left(\mathrm{k}_{\mathrm{o}}\right)$, cue relevance and cue adaptation being held constant, as follows:

$$
\mathrm{E} \sim 1 / \mathrm{k}_{\mathrm{o}}
$$

He proposed, further, that the absolute threshold depended on the angular separation $(\phi)$ of the form $k_{0}=r+s \phi(r, s=$ constants $)$ to yield

$$
E \sim 1 /(r+s \phi) .
$$

Brown (1961) reported that increasing lateral separation increases the differential speed threshold for two stimuli, and Gogel found that "the relative motion cue between more adjacent points was more effective than the relative motion cue between more separated points"' (Gogel, 1974, p. 425). Finally, Harvey and Michon stated that a decrease in angular separation lowered thresholds for "the motion of one spot of light relative to a stationary spot" (Harvey \& Michon, 1974, p. 317). If one assumes that suprathreshold movement parallax is less effective in an experimental situation where the threshold is raised, these studies suggest that the effectiveness of movement parallax decreases with increasing angular separation, the investigation of which is the aim of Experiment 1.

Although an influence of background texture upon the perception of absolute depth of a single stimulus by monocular movement parallax has been found by Ferris (1972), his data are limited by the fact that his subjects seemed to respond equally well to a background and a nonbackground condition in 
which "there was enough noticeable texture ... to permit maximal performance"' (Ferris, 1972, p. 261). Therefore, the relative effect of a visible vs. a nonvisible background was studied in Experiment 1 as well, permitting the determination of a possible interaction between the angular separation of the two objects and the two background conditions.

The purpose of the second investigation was the determination of the influence of background distance on the effectiveness of motion parallax. Our analysis is based on the well-established result of electrophysiological investigations (Barlow \& Hill, 1963a, 1963b, 1964) of motion detection cells in the visual system. Nakayama and Loomis (1974) have, furthermore, suggested a physiological mechanism that "operate(s) on the optical flow over the retina" and "structure(s) the visual field in terms of distinct surfaces" (Nakayama \& Loomis, 1974, p. 63). Depth perception by monocular movement parallax has been shown in another experiment (Hell, 1977) to depend on retinal image motion. Successively disparate images are not sufficient to produce the impression of depth.

If one assumes that motion-sensitive cells are responsible for the processing of movement parallax information as well, it is of interest to know whether movement parallax is a point-to-point effect, i.e., only acting between the retinal points (areas) which the objects under question fall onto, irrespective of the surrounding motion pattern, or if movement parallax is influenced by the motion pattern on adjacent retinal areas, as is the perception of motion. This can be investigated by varying the motion contrast between the objects and the background. If the assumption of a point-to-point effect is valid, this variable is expected to show no influence on depth perception by movement parallax, or vice versa.

Expressed in geometrical terms, the differential angular velocity $\left(\omega_{\mathrm{ab}}\right)$ between two objects, at constant absolute distances $a, b$, from the observer, is independent of background distance $(h)$,

$$
\omega_{\mathrm{ab}}=\mathrm{v}\left(\frac{1}{\mathrm{a}}-\frac{1}{\mathrm{~b}}\right),
$$

$v$ being the velocity of the observer's head movement; the angular velocities of the objects relative to the background,

$$
\omega_{\mathrm{ah}}=\mathrm{v}\left(\frac{1}{\mathrm{a}}-\frac{1}{\mathrm{~h}}\right), \omega_{\mathrm{bh}}=\mathrm{v}\left(\frac{1}{\mathrm{~b}}-\frac{1}{\mathrm{~h}}\right),
$$

vary with the variation of the absolute distance $h$ of the background, as does the ratio $\omega_{\mathrm{ah}} / \omega_{\mathrm{bh}}$ between them. For the derivation of this formula and its underlying assumptions, see Hell (1977).

Experiment 2 was therefore designed to vary selectively the motion contrast between the objects and the background by variation of the background distance, the proximal pattern being indistinguishable for the motionless observer.

\section{METHOD}

Both experiments provided the observer with a display of two objects in front of a luminous background, and required him to match the width of the farther object to the width of the nearer one. The matched width, as in previous experiments (Hell, 1977), was considered a measure of the effectiveness of movement parallax. When no cues to depth are available, the perceived size of objects is determined by the retinal image sizes (law of the visual angle). A control experiment assured that no cues to depth except movement parallax were effective for the monocular observer. In a full cue situation, on the other hand, objects of equal physical size are perceived over a wide range as being equally large, irrespective of their different distances (size constancy law). Thus matched size obtained in a limited cue situation can be regarded as an indicator of the effectiveness of the available cue or cues. In this case, where the more distant of two objects was size-matched to the nearer one, the smaller (larger) the matched variable object, the greater (smaller) the effectiveness of movement parallax. In Experiment 1, the angular separation was varied with and without a textured background being present, and in Experiment 2, the background distance was varied, but the proximal texture remained unchanged.

\section{Subjects}

Ten paid observers ( 5 female, 5 male, mostly students aged from 18 to 32) participated in both experiments. The visual acuity of their dominant eye was at least $20 / 20$. The results of an 11 th observer, whose values were nearly randomly distributed, revealing hardly any depth perception under the conditions of these experiments, were eliminated.

\section{Apparatus}

The apparatus (Figure 1) was designed to provide the observer with a visual field of two black rectangular objects in front of a bright background. The observer sat on an adjustable chair in front of a 13-cm-wide and 1-cm-high slit (SI) and bit into a biteboard whose position could be adjusted so that the observer's dominant eye was vertically centered on the slit at a distance of $5 \mathrm{~cm}$. With the force of .3 newton, the biteboard could be moved laterally parallel to the slit. The amount of movement could be restricted by means of stops. The slit was closed by an electromechanical shutter (Sh) that opened at the start of each measurement and closed after $20 \mathrm{sec}$. At a distance of $33 \mathrm{~cm}$ from the eye, an opaque screen $(\mathrm{Sc})$ limited the angular size of the visual field to $4.3^{\circ}$ vertically and $20.7^{\circ}$ horizontally.

The objects $(\mathrm{Ob})$ consisted of thin black plastic plates bevelled at the edges. Their height was $30 \mathrm{~cm}$ and they were fixed on movable supports so that neither the upper nor the lower limitation was visible to the observer. The standard object was $10 \mathrm{~cm}$ wide. The comparison object consisted of three plates, two of them sliding over the middle one so that its total width could be varied from 8 to $12.3 \mathrm{~cm}$ either by the experimenter or by the observer with the help of a turning knob connected to a flexible cable leading to the object. In both experiments, the standard was placed at $100 \mathrm{~cm}$ and the variable object at $115 \mathrm{~cm}$. They were placed so that at each angular separation they had the same distance from the eye and were perpendicular to the line of sight, the observer's eye being positioned at the center of the slit.

The background $(\mathrm{Bg})$ consisted of stretched translucent paper ( $200 \mathrm{~cm}$ wide, $50 \mathrm{~cm}$ high) rear illuminated by four fluorescent lamps. For all background distances, the lamps were at the same distance from the eye so that the background had the same visual brightness (illuminance $14 \mathrm{~lm} / \mathrm{m}^{2}$ at $195 \mathrm{~cm}$ distance). The ceiling, the table holding the objects, and the backside of the screen were 

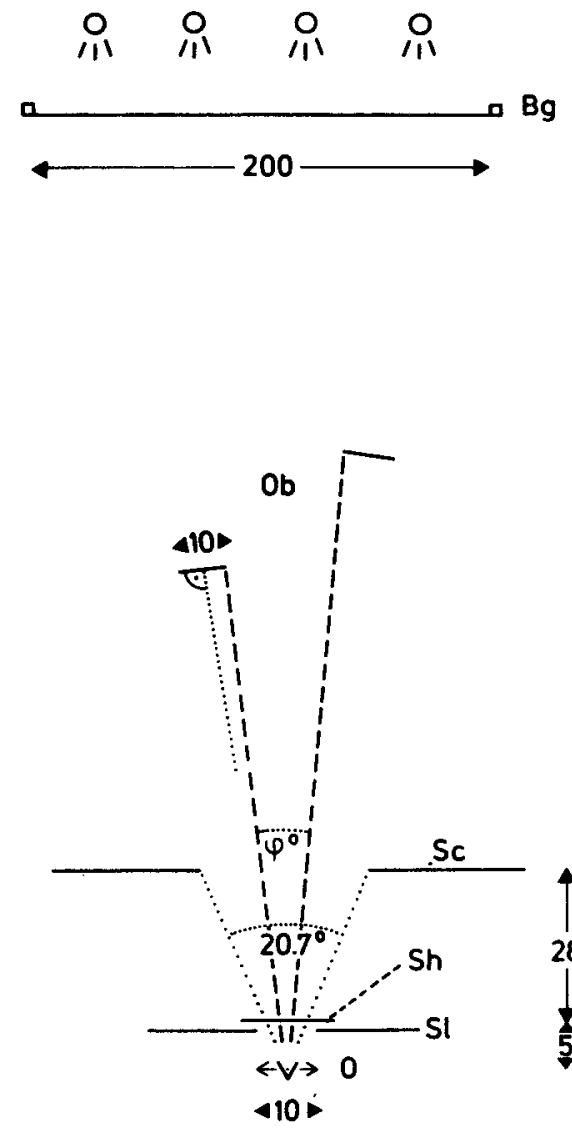

Figure 1. Schematic top-view drawing of the experiment, showing observer (O), slit (SI), shutter (Sh), screen (Sc), objects (Ob), and background $(\mathrm{Bg})$. All distances are given in centimeters.

covered with black cloth and velvet to prevent stray light. The experimenter was careful always to make the same experimental noises between measurements. Two control experiments (described by Hell, 1977) were made to assure that no auditory or visual cues to depth except movement parallax were effective for the monocular observer.

\section{Procedure}

The naive observer was informed about movement parallax and that it "might contribute to the perception of depth differences." His nondominant eye, determined both by the subject's own report and by having him look through a tube, was occluded with an eyepatch. Then eye height and the track of movement were adjusted. The dark adaptation period lasted at least $5 \mathrm{~min}$. The first eight settings after initiating or resuming the experiment after dark adaptation as well as the first four settings after every change of the experimental conditions without dark adaptation were not recorded, in both cases without the observer's knowledge.

The observer was told that he would see two objects and that his task was "to vary the width of the variable object so that you perceive both objects as being subjectively equally wide." He then was given practice in the use of the apparatus as long as he asked for it. Before each measurement, he was told ("left," "right") which object would be variable. The time between measurements was about $1 \mathrm{~min}$. The position (left, right) of the objects and the initial width of the variable object $(9.1,9.7,10.3,10.9)$ were randomly varied, with the restriction that each combination occur twice for each experimental condition to yield a total of
16 measurements per data point for each of the 10 observers. The succession of the experimental conditions was randomly varied among the observers for both experiments. After the first half of each experiment, the order was reversed for each observer. Five observers first participated in Experiment 1; the other five participated first in Experiment 2. The recorded variable was the matched width of the variable (rear) object. In addition, the average velocity of head movement was recorded to ensure a regular performance of the observers. Whenever the observer was not ready or not contented with his final adjustment after the 20-sec trial time or in case of apparatus failure, the measurement was repeated and recorded after the next trial without the observer's knowledge.

Experiment 1. Four different angular separations $\left(4^{\circ}, 8^{\circ}, 12^{\circ}\right.$, $\left.16^{\circ}\right)$ were presented with two different backgrounds. The value for the angular separation refers to the visual angle between the inner limitations of the objects, when the variable was $10 \mathrm{~cm}$ wide and the eye was horizontally centered on the slit. The first background consisted of translucent paper without visible texture. In the second condition, the paper was regularly patterned with black dots of $.4-\mathrm{cm}$ diam and separated by $3 \mathrm{~cm}$ center-to-center. Both backgrounds were placed at $195 \mathrm{~cm}$ distance from the observer's eye. The amount of permissible head movement was $10 \mathrm{~cm}$, and the velocity of head movement was "ad lib."

Experiment 2. The objects were presented with two different background distances $(123,164 \mathrm{~cm})$ under two "movement" conditions (movement parallax, head fixed). The background for the $164-\mathrm{cm}$ distance was the textured background from Experiment 1. The background for the $123-\mathrm{cm}$ condition consisted of the same translucent paper regularly patterned with dots of $.3-\mathrm{cm}$ diameter separated by $2.25 \mathrm{~cm}$ center-to-center, in order to equate the static retinal image of the two backgrounds. The condition "head-fixed" served as a control that both backgrounds presented the same perceptual situation for the observer. Under this condition, the amount of head movement was reduced to zero. The condition "movement parallax" allowed $10-\mathrm{cm}$ head movement, and again the velocity of head movement was "ad lib." Angular separation of the objects was $4^{\circ}$.

\section{RESULTS}

\section{Experiment 1}

The results of Experiment 1 are plotted in Figure 2. A three-way analysis of variance (Separation by Texture by Subjects) results in a significant influence of the angular separation of the objects, $F(3,27)=12.1, p<.001$. The interaction of the angular separation with the observers is insignificant, $F(27,27)=1.5$. The larger the angular separation, the less effective is movement parallax as a cue to depth, as expected. This effect appears for all observers, as shown by the insignificant interaction. The results under the two background conditions differ significantly, $F(1,9)=5.9, p<.05$, as does the interaction of background conditions with the observers, $F(9,27)=11.8, p<.001$. It can be concluded that, with a textured background, movement parallax between two objects is less effective on the average, although two observers showed the opposite effect. This could be attributed either to the pseudorandom succession of conditions, that were balanced for the sample of observers but not for a single observer, or perhaps to a different strategy of the observer with reference to movement parallax. Further- 


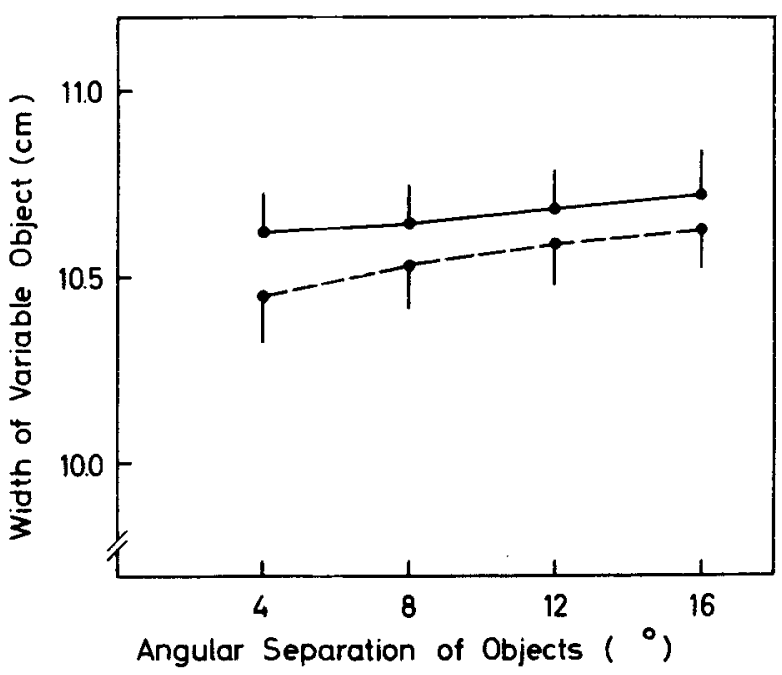

Figure 2. Dependence of the matched width of the variable (rear) object (standard object width $10 \mathrm{~cm}$ ) upon the angular separation of two objects. The upper curve results from measurements with textured background, the lower (dashed) curve from measurements with a background without visible texture. The standard errors are indicated.

more, the interaction of background conditions with the angular separation is insignificant, $F(3,27)=$ 1.7. The influence of angular separation, therefore, is the same under both background conditions. Finally the average velocity of head movement does not differ significantly between the condition with textured background $(9.0 \mathrm{~cm} / \mathrm{sec})$ and without $(9.3 \mathrm{~cm} / \mathrm{sec})$, suggesting that the response differences under the two conditions are attributable to the background pattern rather than to the observer's motor behavior.

\section{Experiment 2}

Figure 3 shows a plot of the data of Experiment 2. A three-way analysis of variance (Distance by Texture by Subjects) reveals that the influence of the background distance (movement parallax and fixed head together) is insignificant, $F(1,9)=1.8$, but that the interaction of background distance with "motion-state" condition is significant, $F(1,9)=$ $9.2, p<.05$. Since the head-fixed condition was considered a control and therefore was expected to yield zero slope, the significant interaction shows that the background distance had an effect on the movement parallax condition. The average velocities of head movement were $9.6 \mathrm{~cm} / \mathrm{sec}(123 \mathrm{~cm}$ background distance) and $8.5 \mathrm{~cm} / \mathrm{sec}(164 \mathrm{~cm}$ background distance). Since these values do not differ significantly, and the effectiveness of movement parallax is nearly independent of the velocity of head movement in this velocity range (see Hell, 1977), the different performance can be attributed to the different background distances, and it therefore can be concluded that movement parallax is more effective for a smaller distance of the textured background.

\section{DISCUSSION}

Lateral separation (Experiment 1), as expected, had an influence on the effectiveness of movement parallax as a cue to depth, as well as the selective variation of motion contrast between objects and background (Experiments 1 and 2). It can be concluded from the second result that movement parallax is not a point-to-point effect acting only between the two retinal areas which the objects under question fall onto, but is influenced by the surrounding retinal motion pattern. Velocity or motion contrast has been shown to be effective (as is brightness contrast) by psychophysical (Loomis \& Nakayama, 1973; MacKay, 1976; Tynan \& Sekuler, 1975) and electrophysiological research (Bridgeman, 1972; Walker \& Powell, 1974). This suggests that both motion perception and depth perception by movement parallax are based on the same mechanism, i.e., on the analysis of the retinal motion pattern by motion detectors, and a possible common explanation of the results of both experiments can be thought of as follows.

It is postulated that whenever the detectability of absolute (relative) motion is improved, i.e., the threshold for motion detection is lowered, the

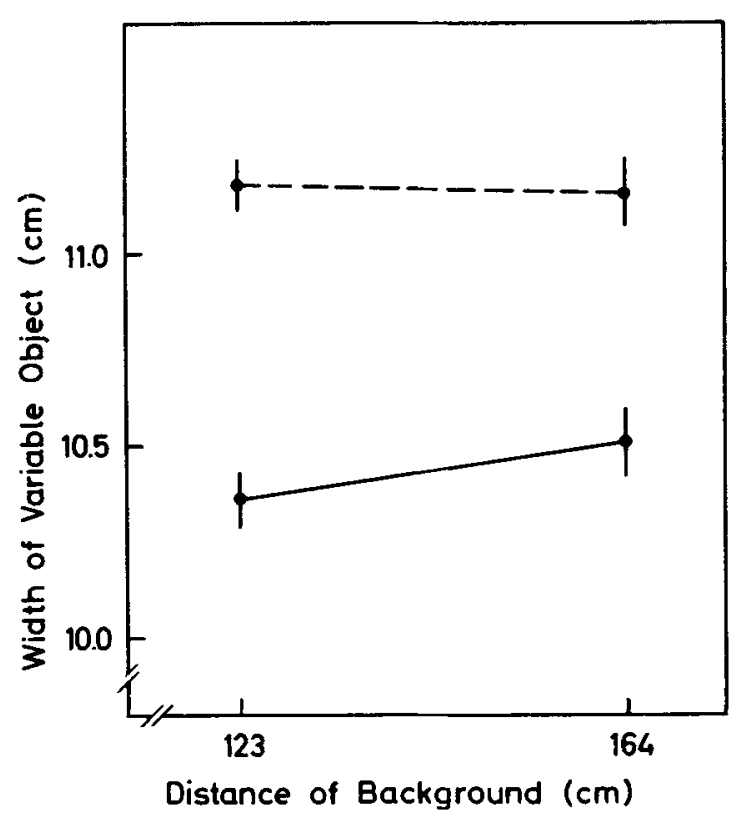

Figure 3. Dependence of the matched width of the variable (rear) object (standard object width $10 \mathrm{~cm}$ ) upon the distance of the textured background from the eye. The upper (dashed) curve results from measurements with head fixed, the lower from measurements under movement parallax. The standard errors are indicated. 
effectiveness of absolute (relative) movement parallax as a cue to depth is enhanced.

This postulate predicts that movement parallax effectiveness decreases with increasing angular separation, since it is known that an increase of lateral separation raises the threshold for the perception of relative motion of two dots (objects) (Brown, 1961; Harvey \& Michon, 1974; Zegers, 1948). There are hardly any data to confirm the hypothesis in the case of motion contrast. Leibowitz (1955) found that at long exposure durations "the presence of reference lines lowers the threshold velocity" of a target (Leibowitz, 1955, p. 829). But the situation with relative movement between two objects, as in movement parallax with two objects at different distances, is different.

The above postulate demands, in order to explain the experimental results of this study, that the threshold for the perception of relative motion is lowest without any background reference and increases with increasing motion contrast between the objects and the background, i.e., with increasing velocity of the background relative to the objects. This hypothesis seems reasonable but demands experimental support beyond the present study.

\section{REFERENCE NOTE}

1. Eriksson, E. S. Movement parallax and distance perception. Report 117. University of Uppsala, Uppsala, Sweden, 1972.

\section{REFERENCES}

Barlow, H. B., \& Hill, R. M. Evidence for a physiological explanation of the waterfall phenomenon and figural after-effects. Nature, 1963, 200, 1345-1347. (a)

Barlow, H. B., \& Hill, R. M. Selective sensitivity to direction of movement in ganglion cells of the rabbit retina. Science, 1963, 139. 412-414. (b)

BARLOW, H. B., \& HILL, R. M. Retinal ganglion cells responding selectively to direction and speed of image motion in the rabbit. Journal of Physiology, 1964, 173, 377-407.

Bridgeman, B. Visual receptive fields sensitive to absolute and relative motion during tracking. Science, 1972, 178, 1106-1108.

Brown. R. H. Visual sensitivity to differences in velocity. Psychological Bulletin, 1961, 58, 89-103.
Ferris. S. H. Motion parallax and absolute distance. Journal of Experimental Psuchology, 1972, 95, 258-263.

FreEman, R. B., JR. Theory of cues and the psychophysics of visual space perception. Psychonomic Monograph Supplement, 1970. 3. 171-181.

Gibson, E. J., Gibson, J. J., Smrrh, O. W., \& Flock, H. Motion parallax as a determinant of perceived depth. Journal of Experimental Psychologv. 1959, 58. 40-51.

GOGEL, W. C. Relative motion and the adjacency principle. Quarterly Journal of Experimental Psychology, 1974, 26, 425.437.

Gogel, W. C., \& TIETz, J. D. Absolute motion parallax and the specitic distance tendency. Perception \& Psychophysics. 1973. 13. 284-292.

Gogel. W. C., \& Tietz. J. D. The effect of perceived distance on perceived movement. Perception \& Psychophysics, 1974, 16, 70-78.

Graham, C. H., Baker, K. E., Hecht, M.. \& Lloyd, V. V. Factors influencing thresholds for monocular movement parallax. Journal of Experimental Psychology, 1948, 38, 205-223.

Harvey, L. O., \& Michon, J. A. Detectability of relative motion as a function of exposure duration, angular separation, and background. Journal of Experimental Psychology. 1974, 103. 317-325.

HELL, W. Movement parallax: An asymptotic function of amplitude and velocity of head motion. Vision Research, 1977, in press.

JoHansson, G. Monocular movement parallax and near-space perception. Perception, 1973, 2, 135-146.

LEIBOWITZ. H. W. Effect of reference lines on the discrimination of movement. Journal of the Optical Society of America, 1955, 45. 829-830.

Loomis, J. M., \& NakAYama, K. A velocity analogue of brightness contrast. Perception. 1973, 2, 425-428.

Mackay. D. M. Perceptual conflict between visual motion and change of location. Vision Research, 1976. 16. 557-558.

Nakayama, K., \& Loomis, J. M. Optical velocity patterns, velocity-sensitive neurons, and space perception: A hypothesis. Perception, 1974, 3, 63-80.

Smith, O. W., \& SMith, P. C. On motion parallax and perceived depth. Journal of Experimental Psychology, 1963, 65, 107-108.

Tynan, P.. \& Sekuler, R. Simultaneous motion contrast: Velocity, sensitivity and depth response. Vision Research, 1975, 15. 1231-1238.

WALker, P. \& P Powell, D. J. Lateral interaction between neural channels sensitive to velocity in the human visual system. Nature. 1974, 252, 732-733.

ZEGERs, R. T. Monocular movement thresholds as functions of field size, field position, and speed of stimulus movement. Journal of Psychology, 1948, 26. 477-498.

(Received for publication December 7, 1976; revision accepted July 28.1977. ) 